\title{
Medición del riesgo suicida, bienestar psicológico y estructura familiar en estudiantes de primer ingreso a las carreras de la salud ${ }^{1}$
}

Determine the suicidal risk, as well as psychological well-being and the family structure of young new entrants to the health careers.

\author{
Luz de Lourdes Eguiluz R. ${ }^{2}$, Juan Mera M. ${ }^{3}$ \\ Fes-Iztacala, Universidad Nacional Autónoma de México \\ (RECIBIDO 02/01/2014, ACEPTADO 24/03/2014)
}

\begin{abstract}
RESUMEN
El objetivo de esta investigación es conocer el riesgo suicida, el bienestar psicológico y relacionar estas dos variables con la estructura familiar de los jóvenes de nuevo ingreso a las carreras de Salud. Se evaluó con tres instrumentos: Escala de evaluación de la cohesión y adaptabilidad familiar (Faces IV), el inventario de riesgo suicida y la escala de bienestar psicológico subjetivo. Los resultados señalan respecto a la estructura familiar que el $87 \%$ piensa que su familia es caótica y el $28 \%$ considera que su familia no está cohesionada. El resultado del IRIS mostró que 8 de los 329 fueron identificados con alto riesgo suicida, 11 con alto riesgo de depresión y 10 alumnos carecen de factores que pueden protegerlos de los riesgos a los que están expuestos. Por último, en la percepción sobre su bienestar en 71 de los 329 jóvenes es muy bajo, por lo que se encuentran en posibilidad de sufrir depresión o incluso estar en riesgo suicida.
\end{abstract}

Palabras clave: riesgo suicida, bienestar subjetivo, estructura familiar, jóvenes, licenciaturas salud.

\begin{abstract}
The purpose of this research is to determine the suicidal risk as well as psychological well-being and relate these two variables with the family structure of young new entrants to the Health Careers. This was assessed with three instruments: Family Adaptability and Cohesion Evaluation Scale (FACES IV), the IRIS for suicide risk, and the Subjective and Psychological Well-Being Scale. Results show, respect to the family structure, that $87 \%$ of the participants think that their family is chaotic and $28 \%$ consider that their family is not
\end{abstract}

1 Esta investigación se hizo gracias el apoyo del Proyecto de Investigación PAPIME 304313

2 La Dra. Eguiluz es jefa del proyecto PAPIME 304313, Prof Titular "C" TC. Definitivo en la Licenciatura en Psicología y Tutora del Posgrado en la FES-Iztacala de la Universidad Nacional Autónoma de México. Email. eguiluz@unam.mx

3 El psicólogo Mera es egresado de la carrera de Psicología y forma parte del Proyecto de Investigación PAPIME 304313, también en la FES-Iztacala, UNAM 
MEDICIÓN DEL RIESGO SUICIDA, BIENESTAR PSICOLÓGICO Y ESTRUCTURA FAMILIAR EN ESTUDIANTES DE PRIMER INGRESO...

cohesive. Results of the IRIS showed that eight of the 329 were identified with high suicidal risk, eleven with high risk of depression and ten students lack of factors that can protect them from the risks to which they are exposed. Finally, the perception of their well-being in 71 of the 329 young people is very low, so they are found in possibility of depression or even being at risk of suicide.

Keywords: suicide risk, subjective well-being, family structure, youth, health bachelor degree.

\section{INTRODUCCIÓN}

Se considera "comportamiento suicida" desde la ideación en sus diferentes expresiones, pasando por las amenazas, los gestos e intentos, hasta el suicidio propiamente dicho. La presencia de cualquiera de estos comportamientos, ya sea de forma esporádica o de manera frecuente, debe considerarse como un signo de alto riesgo para la persona que lo presenta (Eguiluz, 2010).

El suicidio es considerado en México como una problemática de salud pública, ya que en la actualidad es una de las cinco causas de muerte entre los grupos etarios de 15 a 34 años y la tercera entre los jóvenes de 15 a 24 años (Borges, Orozco, Benjet \& Medina-Mora, 2010). El suicidio de un individuo afecta a una gran cantidad de personas, porque lo que pudiera parecer una decisión personal que afecta exclusivamente a quien lo realiza conmueve a toda la red social de pertenencia.

Es difícil determinar la tasa de intento de suicidio que existe en nuestro país, porque existe un subregistro debido a que muchos intentos no son reportados o incluso algunos otros se ocultan de forma intencional; sin embargo, se estima que por cada suicidio consumado, hay 18 intentos de suicidio (García, Sahagún, Ruiz, Sánchez, Tirado, \& González, 2010).

De acuerdo con Borges et al. (2010) hay 4.8 suicidios masculinos por 1 femenino. La edad en que se ha reportado la mayor tasa de intento suicida, $4.26 \%$, es de los 12 a 17 años, seguida por $3.75 \%$ en las edades de 18 a 29 años y, por último, con el $2.61 \%$ en las edades entre 30 y 65 años. De estos datos se deduce que la población que se encuentra en mayor peligro son los adolescentes y que el riesgo va disminuyendo a medida que se avanza en edad. Esta tendencia se observa de manera muy clara para las mujeres y menos marcadamente para los hombres. Típicamente, $25 \%$ de quienes intentaron una vez el suicidio lo intentará nuevamente dentro del siguiente año y 10\% lo logrará en el plazo de diez años (Gutiérrez, Contreras \& Orozco, 2006).

El bienestar psicológico subjetivo (BPS) es concebido como resultado de la integración de dos tradiciones conceptuales: una tradición de tendencia hedónica referida al "bienestar subjetivo" y otra de tendencia eudamónica referida al "bienestar psicológico” (Díaz, Rodríguez, Blanco, Moreno, Gallardo, Valle y Van 
Dierendonck, 2006). Es así que con base en esta perspectiva integradora del BPS son reconocidos sus componentes hedónicos: la satisfacción vital y la afectividad positiva en el momento presente; y sus componentes eudamónicos: la búsqueda del crecimiento personal, el desarrollo del potencial humano y el sentido de la vida en el presente y en el futuro (significado prospectivo).

Los esfuerzos por comprender el bienestar y sus causas no son nuevos, a lo largo de la historia siempre ha suscitado interés (McMahon, citado en Vázquez, Hervás, Rahona y Gómez, 2009). En cierto sentido, todas las intervenciones -médicas, psicológicas, pero también políticas, sociales o económicas- tienen como uno de sus objetivos principales aumentar la calidad de vida de las personas (Vázquez, op. cit., 2009). De hecho, muchas de las decisiones que tomamos cotidianamente son efectuadas sopesando el nivel de felicidad que alcanzaremos nosotros o nuestros seres más queridos (Gilbert, citado en Vázquez, op. cit., 2009).

En la declaración final de la primera reunión de la OMS sobre promoción de la salud, celebrada en Ottawa en 1986, se afirmaba que: "la salud es un estado completo de bienestar físico, mental y social y no meramente la ausencia de enfermedad o de minusvalía. La salud es un recurso de la vida cotidiana, no el objeto de la vida. Es un concepto positivo que subraya los recursos sociales y personales, así como las capacidades físicas" (WHO, 1986, Ottawa Charter for Health Promotion).

Recientemente Myers, McCollam \& Woodhouse (2005), definieron la salud mental como "la resiliencia mental y espiritual que nos permite disfrutar de la vida y sobrevivir al dolor, la decepción y la tristeza. Es un sentimiento positivo de bienestar y una creencia subyacente en uno mismo y en la propia dignidad y la de los demás".

Por otra parte, diversas escuelas de terapia familiar han planteado reiteradamente la necesidad de instrumentos adecuados que permitan estudiar y valorar el funcionamiento familiar. Uno de los modelos con mayor relevancia actualmente en la comprensión de los sistemas familiares es el modelo circumplejo, desarrollado por Olson, Sprenkle y Russell (1979), intentando estructurar el confuso panorama conceptual del momento. Teniendo como respaldo teórico este modelo, se desarrolló el Family Adaptability and Cohesion Evaluation Scale (Faces) en 1980, que se ha utilizado y confiabilizado en población mexicana y española (Martínez, Iraurgi, Galíndez \& Sanz, 2006; Costa, González, Masjuan, Trápaga, Del Arca, Scafarelli, \& Feibuscheurez, 2009).

El modelo circumplejo está compuesto por tres dimensiones: flexibilidad, cohesión y comunicación. Cada una de estas dimensiones está compuesta por diferentes variables que contribuyen a su comprensión. Los conceptos vinculados con la cohesión son: relaciones e implicación familiar, coaliciones padres-hijos, límites internos y externos. Mientras que los conceptos vinculados con la flexibilidad son: liderazgo, disciplina, negociación, roles y reglas familiares. Tanto la cohesión como la adaptabilidad son curvilíneas, esto implica que ambos extremos de las dimensiones son disfuncionales, siendo los niveles intermedios los relacionados con un buen funcionamiento familiar. 
Debido al alto riesgo suicida que se presenta en los adolescentes y siendo la universidad una institución dedicada a la promoción de la salud en la que su población por estos hechos no está exenta de riesgo, consideramos de gran importancia realizar una evaluación en los estudiantes de nuevo ingreso en las siguientes carreras de la salud: Enfermería, Odontología y Psicología, con el objeto de conocer el índice de prevalencia de ideación suicida, de modo que, teniendo estos datos duros, sea factible ofrecer una asesoría psicológica oportuna a aquellos estudiantes que así lo requieran.

Un segundo objetivo es conocer cuáles son las particularidades que podrían conformar una tipología familiar que incremente la probabilidad de presentar ideación suicida, así como conocer los factores protectores que disminuyen o eliminan el riesgo suicida. Es por esto que, además de aplicar el inventario de riesgo e ideación suicida y el cuestionario Faces IV, de los que hemos hablado, consideramos necesario aplicar la escala de bienestar psicológico subjetivo, que nos permitirá conocer los factores de protección existentes en los jóvenes, tan importantes para el tratamiento de emergencia psicológica.

\section{Objetivos de investigación}

A partir de la aplicación de una batería de pruebas se pretende conocer la incidencia/presencia de ideación suicida que existe en los jóvenes de primer ingreso del turno matutino de las carreras de Odontología, Enfermería y Psicología, así como obtener una tipología de la estructura familiar prevaleciente en la muestra estudiantil y conocer el nivel de bienestar psicológico percibido por los estudiantes. Si conocemos estos datos, podemos identificar a los estudiantes que se encuentran en riesgo suicida para ofrecerles el servicio de atención psicológica de emergencia, con la intención de prevenir el suicidio.

\section{MÉTODO}

\section{Tipo y diseño general del estudio}

Se trata de una investigación de corte transversal de una sola medición, cuya finalidad fue conocer la incidencia/presencia que tiene la ideación suicida, además de identificar las estructuras familiares y conocer la percepción de bienestar subjetivo existente en la población estudiantil que está cursando el primer semestre.

\section{Selección y tamaño de muestra}

Se utilizó un modelo probabilístico por conglomerados para elegir a la muestra. Los grupos de primer semestre fueron sorteados, una vez elegidos se habló con el profesor encargado del grupo y se condujo al grupo al salón donde tenían que responder los cuestionarios. El total de la muestra estuvo compuesta por 329 estudiantes de primer semestre del turno matutino, de los cuales 129 estudiantes 
(106 mujeres y 23 hombres) fueron de la carrera de Enfermería, 94 estudiantes (62 mujeres y 32 hombres) de la carrera de Odontología, y 106 estudiantes (78 mujeres y 28 hombres) de la carrera de Psicología. La edad promedio fue de 18.9 años, con una desviación estándar de 2.76.

\section{Procedimientos y características de los instrumentos a utilizar}

El orden de aplicación de las pruebas fue como se describe a continuación: A) La escala de evaluación de la cohesión y la adaptabilidad familiar (Family Adaptability and Cohesion Evaluation Scale, Faces IV), con coeficiente de fiabilidad alfa de Cronbach de 0.70. Es una adaptación y traducción al español (Martínez et al., 2006) del cuestionario original elaborado por David Olson (2000, Olson, Gorall \& Tiesel, 2006, Olson, 2011). Este instrumento identifica la estructura familiar en tres dimensiones: 1) flexibilidad, es decir, la habilidad de la familia para cambiar, 2) cohesión, tiene que ver con el vínculo emocional que los miembros de una familia tienen entre sí y 3) comunicación, que implica el poder hablar, escuchar al otro y respetar las diversas opiniones. B) Se utilizó además la escala de bienestar psicológico subjetivo (EBPS), con fiabilidad de alfa de Cronbach de 0.70. Esta prueba es la adaptación y traducción al español de México (Díaz et al., 2006) del cuestionario Psychological Well-Being (PWB) elaborado por Carol Ryff, en el que se consideran seis variables: 1) autoaceptación, 2) relaciones positivas con los otros, 3) crecimiento personal, 4) propósito de vida, 5) dominio del entorno y 6) autonomía. Por último se empleó también C) el inventario de riesgo e ideación suicida (IRIS) con coeficiente de fiabilidad alfa de Cronbach de 0.95. Este inventario fue elaborado por Quetzalcóatl Hernández y Lucio (2007), el cual evalúa tres dimensiones: 1) ideación suicida, 2) depresión y desesperanza y 3) ausencia de factores protectores.

Para obtener la participación de los alumnos, primero se habló con los jefes de cada una de las carrera seleccionadas, con la intención de explicarles los objetivos del proyecto. Los grupos de nuevo ingreso de cada carrera fueron sorteados y, posteriormente, se visitó a cada grupo y se solicitó al profesor de la materia el día y la hora que podía permitir a su grupo asistir al centro de cómputo para ser evaluado. En el día y hora acordado una persona del proyecto de investigación pasó por el grupo y lo condujo a la sala de evaluación. Para la aplicación de esta batería, se solicitó la sala de cómputo, que cuenta con 30 computadoras para uso de los estudiantes. Una vez que cada grupo terminó de responder las pruebas se recogió de cada máquina la información con los datos de cada alumno, para posteriormente vaciarlos en un programa de Excel diseñado ex profeso.

La batería se aplicó durante 4 días, en el horario matutino de 10:00 a 13:00. Cada aplicación fue de 40 minutos aproximadamente, reuniendo un total de 330 aplicaciones. Las pruebas fueron aplicadas a 10 grupos de alumnos ( 3 de Psicología, 3 de Enfermería y 4 de Odontología) que cursaban el primer semestre en el turno matutino. 
MEDICIÓN DEL RIESGO SUICIDA, BIENESTAR PSICOLÓGICO Y ESTRUCTURA FAMLIAR EN ESTUDIANTES DE PRIMER INGRESO...

\section{RESULTADOS}

Después de haber realizado la aplicación de la batería de pruebas, obtuvimos los siguientes resultados.

El modelo circunflejo de los sistemas familiares (Fases IV) es un modelo dinámico que, como se ha mencionado, integra tres dimensiones que se han encontrado como relevantes en distintas teorías y abordaje clínico en familia. Estas dimensiones son: flexibilidad, cohesión y comunicación.

Su aplicación se considera útil para realizar un diagnóstico relacional, destacando áreas saludables (balanceadas) y problemáticas (desbalanceadas) en los sistemas (familias o parejas) que estudia.

La dimensión de flexibilidad, también conocida como adaptabilidad, incluye la calidad y expresión del liderazgo, la organización, los papeles que juega cada miembro del grupo familiar, las reglas y negociaciones que existen en la familia. En esta dimensión encontramos que el $87 \%$ de la muestra evaluada vive en una familia considerada "caótica", mientras que el $7 \%$ vive en una familia de límites "flexibles". Las familias "estructuradas" componen el $4 \%$ de la muestra y el $1 \%$ de familias "rígidas". El valor "no específico" se refiere a las personas que no contestaron este reactivo en la prueba. Las posiciones intermedias en los niveles centrales (familias estructuradas o flexibles) conlleva un funcionamiento familiar más saludable. Mientras que los extremos, como es el caso, donde los jóvenes consideran que están viviendo en una familia "Caótica" implica un funcionamiento más problemático (figura 1 ).

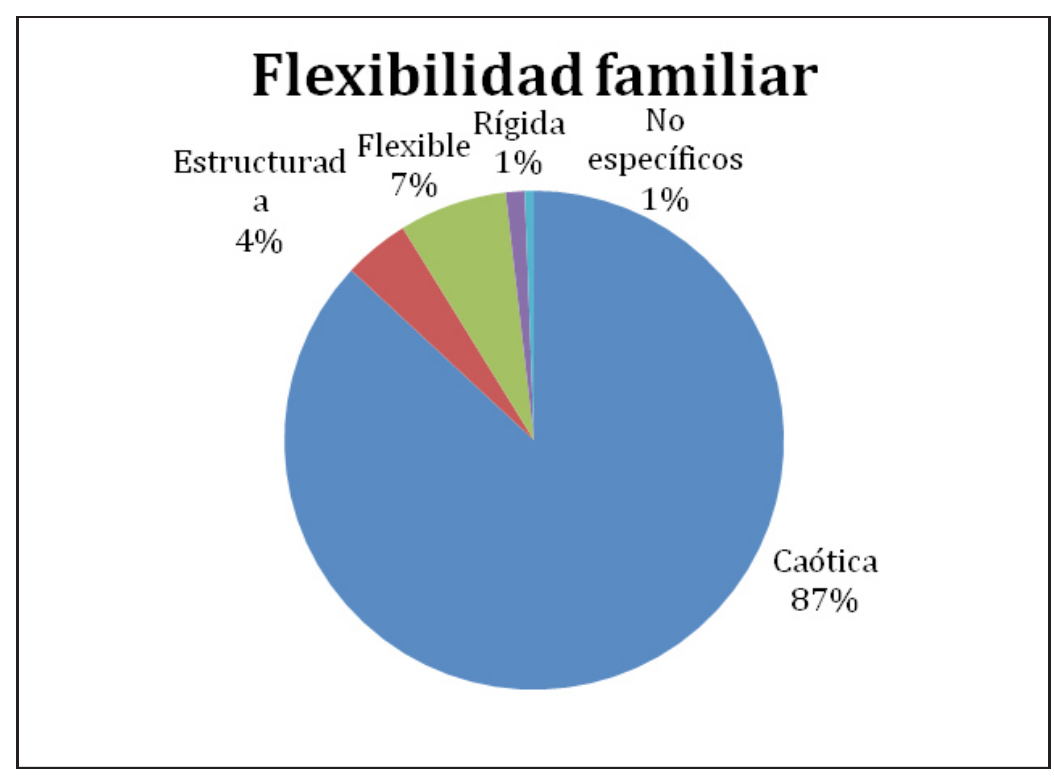

Fig. 1. Tipologías de familias con respecto a su flexibilidad. 
Por otro lado, la dimensión cohesión es definida como los lazos emocionales que los integrantes del grupo familia guardan entre sí. Las hipótesis del modelo señalan que, de las cuatro dimensiones, las familias separadas o las conectadas, que se encuentran en el centro, son las que tienen un nivel de funcionamiento más saludable, mientras que por el contrario los niveles extremos, familias desligadas y enmarañadas, implican un funcionamiento más problemático.

En la muestra estudiada encontramos que el $28 \%$ identifica que su familia es "separada o poco conectada" y el $27 \%$ "relacionada o conectada". Por otra parte, el $24 \%$ de las familias de la muestra se identifica como "desligada" y el $21 \%$ como familia "aglutinada o enmarañada". El valor "no especifico" se refiere a las personas que no contestaron este reactivo en la prueba (figura 2).

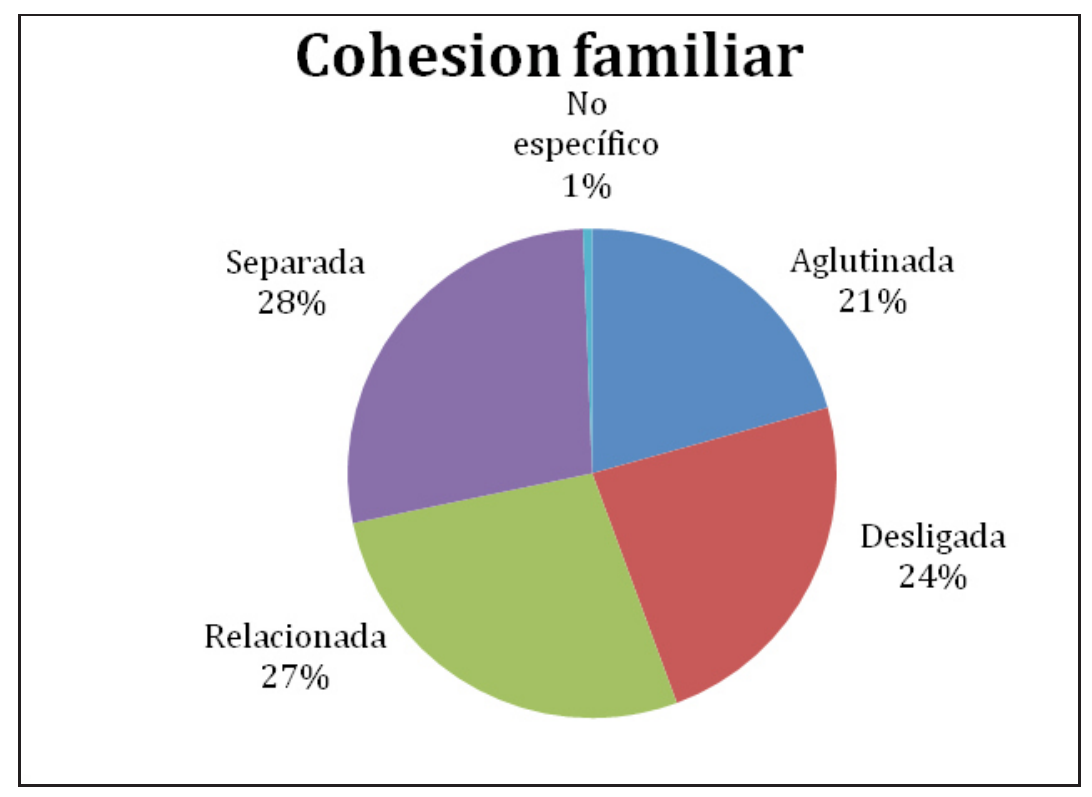

Fig. 2. Tipologías de familias con respecto a su cohesión.

La tercera de las dimensiones -comunicación- no se evalúa directamente, ya que se considera una dimesión facilitadora que permite que se expresen en sus niveles positivos, tanto la flexibilidad como la cohesión. La comunicación tiene que ver con la posibilidad de expresarse, de escucharse, de respetar las opiniones de unos y otros y de compartir los sentimientos.

Respecto a la evaluación realizada con la escala de bienestar psicológico subjetivo (EBPS), pudimos identificar a 2 grupos: los que tienen puntuaciones por encima del promedio y los que tienen puntuaciones inferiores al promedio.

El primer grupo está compuesto por 50 estudiantes que en las 6 dimensiones evaluadas por la EBPS obtienen puntajes por encima del promedio, lo cual significa 
MEDICIÓN DEL RIESGO SUICIDA, BIENESTAR PSICOLÓGICO Y ESTRUCTURA FAMILIAR EN ESTUDIANTES DE PRIMER INGRESO...

que estos jóvenes perciben un alto nivel de bienestar en sus vidas, lo que hace que sean menos vulnerables al riesgo suicida o depresión.

Por otro lado, encontramos 71 estudiantes que en 5 de las 6 dimensiones evaluadas obtuvieron puntuaciones menores al promedio, lo cual significa que su nivel de percepción respecto a su bienestar es muy bajo, por lo que se encuentran en posibilidad de sufrir depresión o incluso estar en riesgo suicida.

\section{DISCUSIÓN}

En la tabla 1 podemos identificar el número de estudiantes que obtuvieron un puntaje menor al promedio en cada una de las dimensiones evaluadas. La importancia de estos resultados radica en que podemos identificar las dimensiones de "dominio del entorno" y "autonomía" como peor percibidas por los jóvenes, es decir, estas dos dimensiones son las que se necesita fortalecer en el $47 \%$ de la muestra para elevar su nivel de bienestar psicológico subjetivo. Sin embargo, podemos ver que las seis dimensiones presentan un porcentaje similar, por lo que es importante que se realicen intervenciones en todas las áreas para elevar el nivel de bienestar psicológico subjetivo en general.

Tabla 1. Número de jóvenes que obtuvieron puntuaciones menores al promedio en cada dimensión del EBPS

\begin{tabular}{ccccccc}
\hline & Autoaceptación & $\begin{array}{c}\text { Relaciones } \\
\text { positivas }\end{array}$ & Autonomía & $\begin{array}{c}\text { Dominio del } \\
\text { entorno }\end{array}$ & $\begin{array}{c}\text { Crecimiento } \\
\text { personal }\end{array}$ & $\begin{array}{c}\text { Propósito } \\
\text { de vida }\end{array}$ \\
\hline Total & $145(44 \%)$ & $145(44 \%)$ & $153(47 \%)$ & $155(47 \%)$ & $142(43 \%)$ & $\begin{array}{c}144 \\
(44 \%)\end{array}$ \\
\hline Mujeres & 110 & 111 & 119 & 113 & 102 & 99 \\
\hline Hombres & 35 & 34 & 34 & 42 & 40 & 45 \\
\hline
\end{tabular}

Con respecto a la evaluación realizada por el IRIS, 8 estudiantes fueron identificados con alto riesgo suicida, 11 con alto riesgo de depresión y 10 alumnos que carecen de factores que pueden protegerlos de los riesgos a los que están expuestos (tabla 2). De estos alumnos, 4 de ellos se encuentran en alto riesgo, debido a que en las tres dimensiones evaluadas por el IRIS presentan puntajes de riesgo y otros 4 se encuentran en riesgo medio, debido a que tienen puntajes de riesgo en dos dimensiones (Tabla 3).

Tabla 2. Alumnos identificados en riesgo por la prueba IRIS

\begin{tabular}{cc}
\hline 8 & Con riesgo suicida \\
\hline 11 & Con riesgo de depresión \\
\hline 10 & En posible riesgo por circunstancias \\
\hline
\end{tabular}


Tabla 3. Tipología de riesgo de alumnos identificados por la prueba IRIS

\begin{tabular}{ll}
\hline 4 & Alto riesgo \\
\hline 4 & Mediano Riesgo \\
\hline 9 & Bajo riesgo \\
\hline
\end{tabular}

Los jóvenes que fueron identificados con riesgo suicida o depresión fueron contactados mediante correo electrónico para ofrecer el servicio de atención psicológica de emergencia. Fueron atendidos por terapeutas con formación en Terapia de Emergencia en la FES Iztacala.

Al relacionar las evaluaciones, identificamos que el $43 \%$ de las familias de aquellos alumnos que están en riesgo suicida consideran que su familia tiene una flexibilidad o adaptabilidad "caótica" (fig. 3) y son categorizados como "separados o no relacionados" en la dimensión de cohesión (fig. 4). Estas características nos llevan a pensar que en estas familias no hay o están desdibujadas las figuras de autoridad, no hay reglas estructuradas y claras, lo que puede dificultar las negaciaciones entre los miembros del grupo.

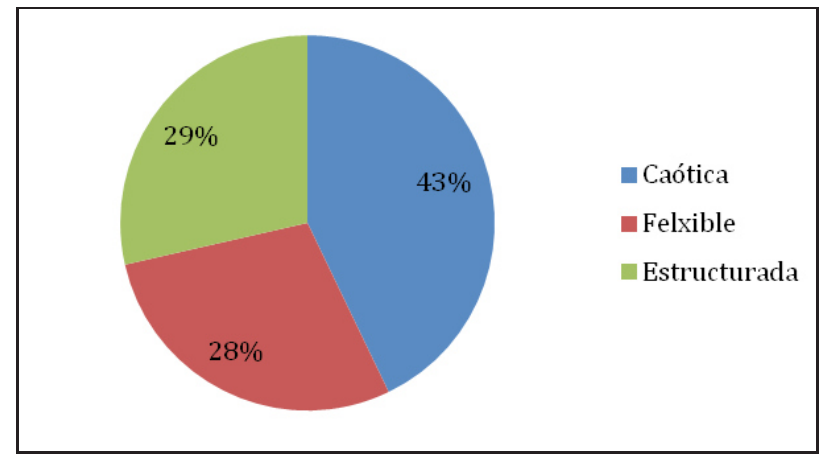

Fig. 3. Flexibilidad de la familia con un miembro en riesgo suicida.

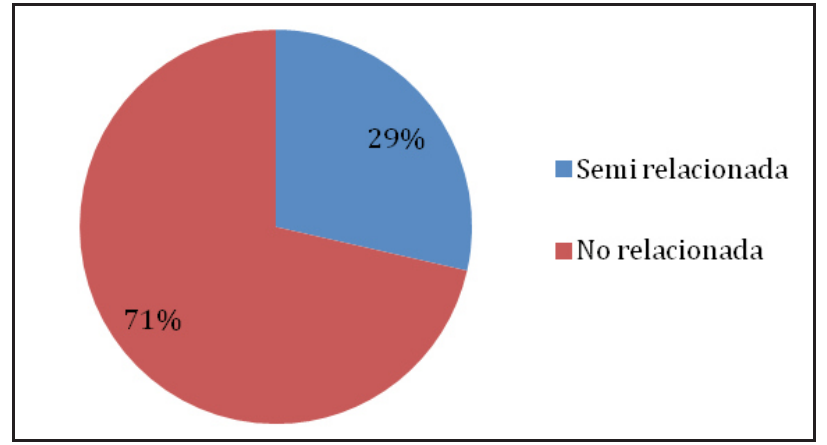

Fig. 4. Cohesión de la familia con un miembro en riesgo suicida. 
MEDICIÓN DEL RIESGO SUICIDA, BIENESTAR PSICOLÓGICO Y ESTRUCTURA FAMILIAR EN ESTUDIANTES DE PRIMER INGRESO...

\section{CONCLUSIONES}

A partir de esto, podemos concluir que la mayoría de las familias de la muestra presentan una flexibilidad "caótica" en un porcentaje bastante alto $(87 \%)$, sin embargo, lo que identifica a las familias de los alumnos en riesgo es que en la dimensión de cohesión son identificadas como "no relacionadas o desligadas". Por ello, se puede decir que una de las áreas de prevención para el riesgo suicida o la depresión estaría enfocada a fomentar las relaciones al interior del grupo familiar, mejorando el vínculo emocional que existe entre los miembros de la familia. Por los datos obtenidos, podríamos señalar que estos jóvenes perciben a su familia con ausencia de reglas y consecuencias pertinentes (premios y castigos) que les permitan saber cómo deben comportarse. No hay un liderazgo claro y objetivo, es decir, ni el padre ni la madre juegan el rol de autoridad (quizá por falta de presencia física y emocional). Los padres no tiene poder para ordenar, organizar, poner límites, lo que dificulta la posibilidad de llegar a acuerdos o de negociar entre ellos. Pero, además, como muestran los resultados en la EBPS, las dimensiones de "dominio del entorno" y "autonomía" son las que son percibidas con los índices más bajos. Aunque habría que reforzar también las seis dimensiones restantes, cuyos porcentajes también se localizan por debajo de la media. Se considera la posibilidad de realizar intervenciones en todas estas áreas para elevar el nivel de bienestar subjetivo, lo que podría contrarrestar los datos correspondientes a la estructura familiar y al riesgo de ideación suicida.

\section{REFERENCIAS BIBLIOGRAFICAS}

Borges, G., Orozco, R., Benjet, C., \& Medina-Mora, M. (2010). Suicidio y conductas suicidas en México: retrospectiva y situación actual. Salud Pública, 292-304.

Costa, B. D., González, T. M., Masjuan, N. Trápaga, M. A., Del Arca, D., Scafarelli, L. \& Feibuscheurez, A. (2009). Escala de evaluación del funcionamiento familiar-FACES IV- Proceso de adaptación a Montevideo, Uruguay. Rev. Ciencia Psicológica 3(1), 43-56

Díaz, D., Rodríguez, R., Blanco, A., Moreno, B., Gallardo, I., Valle, C. \& Van Dierendonck, D., (2006). Adaptación española de las escalas de bienestar psicológico de Ryff. Psicothema, 18(3), 572-577.

Eguiluz, L.L. (2010). ¿Qué podemos hacer para evitar el suicidio México: Pax- México

García, H., Sahagún, J., Ruiz, A., Sánchez, G., Tirado, J., \& González, J. (2010). Factores de riesgo, asociados a intento de suicidio, comparando factores de alta y baja letalidad. Salud Pública, 713-721.

Gutiérrez, A., Contreras, C., \& Orozco, R. (2006). El suicidio, conceptos actuales. Salud Pública, 29(5), 66-74.

Hernández, Q. \& Lucio, E. (2007). Inventario de Riesgo Suicida para Adolescentes: IRIS. Manual para versión 1.0.2. 
Martínez, A., Iraurgi, I., Galíndez, E. \& Sanz, M. (2006). Family Adaptability and Cohesion Evaluation Scale (FACES): Desarrollo de una versión de 20 ítems en español. International Journal of Clinical and Health Psychology, 6(2), 317-338.

Myers, F., Mc Collam, A. \& Woodhouse, A. (2005). National Programme for Improving Mental Health and Well-Being Addressing Mental Health Inequalities in Scotland. Equal minds. Edinburgh: Scottish Executive. Scottish Development Centre for Mental Health.

Olson, D. H. (2000). Circumplex Model of Marital \& Family Systems. Journal of Family Therapy, 22(2), 144-167.

Olson, D. H. (2011). FACES IV and the Circumplex Model: Validation Study. Journal of Marital \& Family Therapy, Vol. 3(1) 64-80

Olson, D.H. Gorall, D.M. \& Tiesel, J. (2006). FACES IV: Development and Validation: Recuperado el 18 abril 2013 en www.facesiv.com Life Innovations. Minnesota.

Vázquez, C., Hervás, G., Rahona, J. \& Gómez, D. (2009). Bienestar psicológico y salud: Aportaciones desde la Psicología Positiva. Anuario de Psicología Clínica y de la Salud, Vol. 5, pp. 15-28.

World Health Organization (1986). Ottawa Charter for Health Promotion: First International Conference on Health Promotion, Ottawa, November 1986. Geneva: WHO. 